\title{
Multi-ROI Association and Tracking With Belief Functions: Application to Traffic Sign Recognition
}

\author{
Mohammed Boumediene, Jean-Philippe Lauffenburger, Jérémie Daniel, Christophe Cudel, and Abdelaziz Ouamri
}

\begin{abstract}
This paper presents an object tracking algorithm using belief functions applied to vision-based traffic sign recognition systems. This algorithm tracks detected sign candidates over time in order to reduce false positives due to data fusion formalization. In the first stage, regions of interest (ROIs) are detected and combined using the transferable belief model semantics. In the second stage, the local pignistic probability algorithm generates the associations maximizing the belief of each pairing between detected ROIs and ROIs tracked by multiple Kalman filters. Finally, the tracks are analyzed to detect false positives. Due to a feedback loop between the multi-ROI tracker and the ROI detector, the solution proposed reduces false positives by up to $45 \%$, whereas computation time remains very low.
\end{abstract}

Index Terms-Credal association, data fusion, multitarget tracking, traffic sign recognition (TSR).

\section{INTRODUCTION}

A LTHOUGH traffic signs are designed to be clearly visible, they can be missed due to driver distraction or sign masking. This can be avoided by using traffic sign recognition (TSR) systems. Indeed, they can inform the driver of a possibly missed traffic sign. Usually, vision-based TSR consists of a detection and a recognition (or classification) step [1]. In each frame, the detection localizes regions of interest (ROIs) that may contain signs for instance based on shape detection [2] using the Hough transform [3] or histograms of oriented gradients (HOG) [4]. The purpose of recognition is to identify the pictograms present in the ROIs, with the help of support vector machines, K-d trees [4], neural networks [5], etc. When detection and recognition are independently processed, TSR systems show known limitations-multiple detections for the same sign, misdetections due to temporal occlusions, and wrong detections, usually called "false positives" - which can be reduced by adding target tracking [6], [7]. Tracking helps to take account of temporal redundant information of the road scene and thus allows TSR systems to track the signs over time [3], [8]. However, although tracking provides obviously substantial benefits, its application to TSR is still unusual, as shown in Møgelmose et al. [1].

Manuscript received August 29, 2013; revised February 14, 2014; accepted April 10, 2014. This work was supported by a PROFAS research fellowship granted by the Algerian and French governments. The Associate Editor for this paper was Q. Ji.

M. Boumediene and A. Ouamri are with the Laboratoire Signaux et Images, Université des Sciences et de la Technologie d'Oran Mohamed Boudiaf, Oran 31000, Algeria (e-mail: boumediene.m@gmail.com).

J.-P. Lauffenburger, J. Daniel, and C. Cudel are with the Laboratoire Modélisation Intelligence Processus et Systèmes (EA 2332), Université de Haute Alsace, Mulhouse 68093, France (e-mail: jean-philippe.lauffenburger@uha.fr).

Color versions of one or more of the figures in this paper are available online at http://ieeexplore.ieee.org.

Digital Object Identifier 10.1109/TITS.2014.2320536
One of the main tasks of target tracking concerns data association. It defines, at time $k$, the relations between the set of perceived objects (targets) and the set of already known ones (tracks). Association methods are usually based on the Bayesian theory. They are either track oriented, such as $(\mathrm{G}) \mathrm{NN}^{1}$ and (J)PDAF ${ }^{2}$ [7], or target oriented, such as $\mathrm{MHT}^{3}$ [9]. Trackoriented methods perform single-frame associations and are mainly based on the a priori known track number. As a consequence, track appearances are not directly managed but can be possible through the computation of some specific measurements (likelihood ratio, observability measurement, etc.) for (J)PDA filters, for instance. MHT tracks objects over time and defers the assignment decision if ambiguities occur. At each time step, a decision tree containing all the hypotheses is updated, and each hypothesis iteratively leads to a new decision tree, requiring important computation time.

Recently, the use of belief functions [10], [11] and the transferable belief model (TBM) [12] for data association has emerged [13]-[19]. One can particularly cite [14] and [15], in which a comparison with Bayesian tracking solutions is presented, and [16] and [20], highlighting the fact that belief functions constitute a suitable and intuitive framework for data and imperfection modeling. They easily deal with the source ignorance and conflict without any assumptions on the sensor error models. This is the formal context of this paper.

This paper focuses on traffic sign detection and tracking. The detection process, which has been recently presented in [21], is based on corner and edge orientation detection in gray-scale images. The corners help to define the candidate positions (i.e., the ROIs) where the presence of signs is to be confirmed, whereas the edge orientation informs about the sign candidate type (triangular, circular, etc.). The detected ROIs are then spatiotemporally tracked using a TBMbased multi-ROI tracking (MRT) algorithm. The MRT considers the ROIs provided by the detector as information to be combined with the known tracks and thus allows their appearance, disappearance, or evolution along time. The aim is to reduce the influence of sensor inaccuracies and false positives while maintaining a good detection rate. For the associated objects, a spatial data fusion using dynamically managed multiple Kalman filters (KFs) [22] both for their tracking and position prediction in the future frames is integrated to enhance detection performance. This MRT provides a global approach managing all targets and tracks whatever the

\footnotetext{
${ }^{1}(\mathrm{G}) \mathrm{NN}$ : (global) nearest neighbor.

2(J)PDAF: (joint) probability data association filter.

${ }^{3}$ MHT: Multihypothesis tracking.
} 


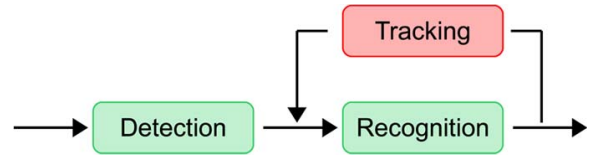

Fig. 1. Basic tracking-based TSR system architecture [1].

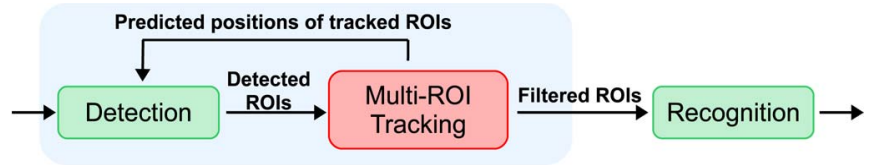

Fig. 2. Overview of the proposed system.

association type (track-to-target, track-to-track, etc.) with a generalized combination rule allowing complexity reduction. In addition, it provides native mechanisms for track initialization, temporary occlusion, and conflicting association management. The system has been implemented in a test vehicle. The results obtained show the benefits in terms of detection rate, false positive reduction and computation time, and satisfactory real-time constraints.

This paper is organized as follows. Section II presents the system overview. The theoretical background of data association in the TBM is presented in Section III. Section IV details the proposed MRT, and its experimental validation is presented in Section V. Finally, Section VI concludes this paper.

\section{System OVERVIEW}

Tracking-based TSR systems are characterized by two architectures: Detection-Recognition-Tracking and DetectionTracking-Recognition. In the first one, the temporal information is used after Recognition (see Fig. 1), so that only the recognized signs are tracked in the future frames. Tracking consequently provides a memory [3], which makes the recognition of the tracked sign in subsequent frames unnecessary [23]. Several approaches have been adopted in the literature for the second architecture, which performs Tracking after Detection. For instance, Fang et al. [24] tracked all detected ROIs until their respective size is sufficiently large for easy recognition, whereas in [25], a classifier separates valid and invalid tracks by using spatiotemporal constraints.

This study adopts a Detection-Tracking-Recognition architecture. The system proposed combines Detection and Tracking in order to deal with temporary occlusions and false positive detections. It is composed of an ROI detector and an MRT algorithm (see Fig. 2). The ROI detection uses corner and edge orientation, which helps to detect signs at the position candidates. This results in the generation of a set of traffic sign candidates (detected ROIs), which can contain false positives. To cope with this problem, the algorithm tracks the detected ROIs to provide filtered ROIs to Recognition. Tracking also feeds back to the detector the predicted position of ROIs (tracked ROIs in Fig. 2) in the subsequent frames. This feedback enhances the detection performance in variable illumination conditions where corner detection can fail. The filtered ROIs represent tracked ROIs with a high confidence score.
This paper focuses on the association and tracking capabilities of the proposed MRT. It will be shown how belief functions provide an interesting framework for the problem description, good association, and tracking performance while reducing the level of false positives.

\section{A. ROI Detection}

Fig. 3 describes the ROI detector. A traffic sign shape detector, which was initially described and evaluated in [21], is implemented here. Singularities or angular edges of traffic signs are detected by the Corner Detection step and represent the position candidates of signs. Fig. 4 shows an example of the position candidates, which are defined by the standard Harris corner detector [26]. For each corner, a candidate ROI is selected according to the shapes in the corner neighborhood. Shape recognition is performed using a coded image, where each pixel is defined according to edge orientations, as depicted in Fig. 5. To recognize triangular shapes, the RANSAC symmetric line detection algorithm from Boumediene et al. [21] is implemented. Its principle is extended with a TemplateMatching process to circular shape detection.

\section{B. Multi-ROI Tracking}

The MRT algorithm is composed of three functions: Filtering, Data Association, and ROI life analysis (see Fig. 6). Filtering performs target tracking by predicting the future position of the tracked ROIs in the frames. Data Association assigns one target provided by the detector to a track and manages the track creation/deletion. ROI life analysis uses the temporal context to validate/reject the tracks transmitted to Recognition. Traffic sign candidates provided by Detection define the targets of the MRT. The tracked ROIs define the tracks to be filtered to reduce false positives. For the track State Estimation, the well-known KF [22] has been adopted as it is one of the most efficient methods. The filter predicts the states of the tracked ROIs from the previous states. These predictions are transmitted to the detector as a priori information to influence future searches. Indeed, predicted positions are added to the position candidates, which are defined by the corner detector. In addition, the filter updates the tracked ROIs states based on the association results.

Data Association consists of three subtasks: Gating, Association, and Track Maintenance. First, Gating eliminates the improbable target-to-track pairings to reduce the computational complexity of Association. This can be done considering, for instance, a distance criterion between the targets and the tracks. Association defines, at time $k$, the relations between targets and tracks. Noisy environments with uncertain measurements make joint association and tracking a challenging task, particularly in multiple target scenarios, when their number is unknown and temporally variable. Uncertainty also occurs when targets are close to each other and, therefore, can fall simultaneously into multiple gates. These conditions involve ambiguities and contradictions in the association since targets could be associated with multiple tracks, and vice versa.

The association is done through an evidential data fusion process to consider and propagate these uncertainties as well 


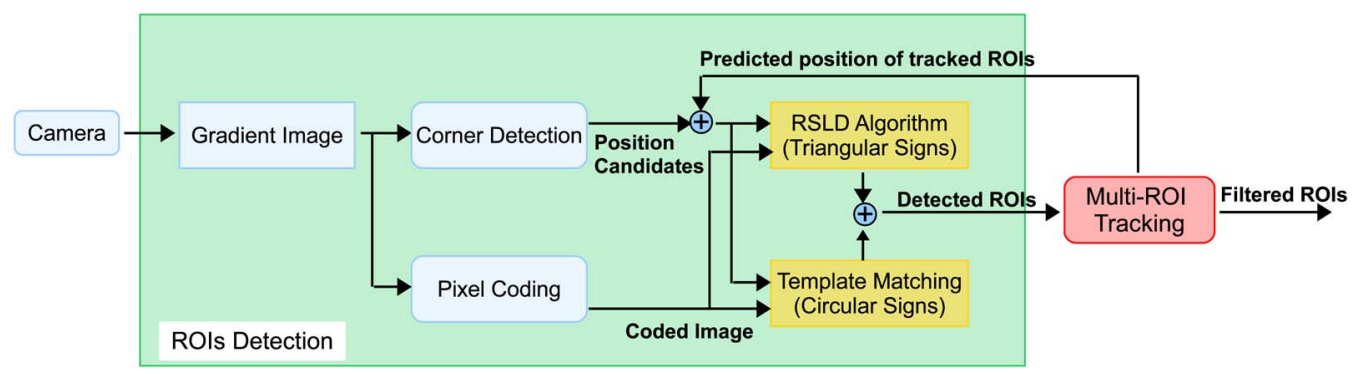

Fig. 3. Block diagram of the ROI detector
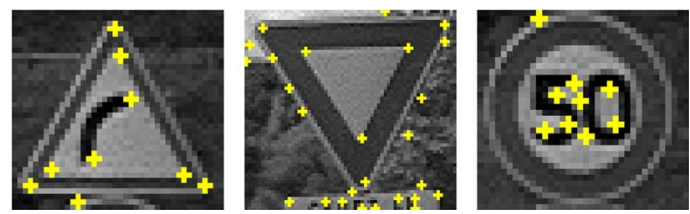

Fig. 4. Examples of detected corners related to traffic signs.

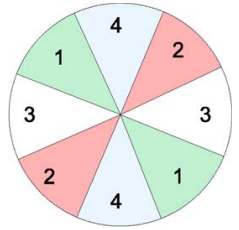

Fig. 5. Segmentation of the orientation space (from [21]). Classes 3 and 4 represent horizontal and vertical pixel edges, respectively. Classes 1 and 2 describe diagonal pixel edges. The non-edge pixel is considered as class 0 .

as sensor imperfections and reduce false detections. Track Maintenance analyzes the selected target-to-track pairs to detect appearances and disappearances. A track appearance/ disappearance leads, respectively, to adding/deleting a KF. However, traffic signs do not suddenly appear and disappear in frames. Therefore, the analysis of the track temporal evolution helps to detect false positives. Indeed, based on the track properties such as its length (number of frames where the track considered is present), a confidence score is defined. A tracked ROI with a low confidence value is considered as a false positive and thus ignored. Otherwise, it is forwarded to the Recognition step. Disappearance is confirmed if no target is assigned to the track several times at a stretch.

The next sections will show that credal association and tracking is able to reduce the false positive rate while keeping a high detection rate. This result is reproducible, whichever traffic sign detector is used.

\section{DATA AsSOCIATION IN THE TBM}

\section{A. TBM: Basic Concepts}

The TBM is a subjective and nonprobabilistic interpretation of the evidence theory [10], [11]. This framework describes a model of uncertain reasoning and decision making based on a credal and a pignistic level. At the credal level, belief masses are used to represent and combine the pieces of information, whereas at the pignistic level, these masses are transformed into probability measurements for decision making.
1) Credal Level: Consider a problem for which all the discrete solutions (also called hypotheses) $H_{j}, j=1,2, \ldots, k$, with $k$ as the number of possible hypotheses, define the frame of discernment $\Theta$, i.e.,

$$
\Theta=\left\{\left\{H_{1}\right\},\left\{H_{2}\right\}, \ldots,\left\{H_{k}\right\}\right\}=\bigcup_{j=1}^{k}\left\{H_{j}\right\} .
$$

$\Theta$ is the ignorance, i.e., the union of all hypotheses. Its corresponding referential subset, a power set denoted $2^{\Theta}$ of $2^{k}$ disjunctions of $H_{j}$, is such that

$$
2^{\Theta}=\left\{\emptyset,\left\{H_{1}\right\}, \ldots,\left\{H_{k}\right\}, \ldots,\left\{H_{1}, H_{2}, H_{3}\right\}, \ldots, \Theta\right\}
$$

where $\emptyset$ represents the impossible hypothesis commonly interpreted as the conflict between sources. A proposition $A=$ $\left\{H_{1}, H_{2}\right\}$ refers to the disjunction " $H_{1}$ or $H_{2}$ ", i.e., either $\left\{H_{1}\right\}$ or $\left\{H_{2}\right\}$ can be the solution to the problem. Each proposition $A$ of $2^{\Theta}$ provided by a source $i$ is characterized by its basic belief mass $(\mathrm{bbm}) m_{i}^{\Theta}(A)$, which represents its veracity

$$
m_{i}^{\Theta}: 2^{\Theta} \rightarrow[0,1], \sum_{A \in 2^{\Theta}} m_{i}^{\Theta}(A)=1 .
$$

When the $k$ answers are exclusive and exhaustive, the solution to the problem is one of the hypotheses of $\Theta$, and a mass on $\emptyset$ is not allowed. Practically, in applications such as target tracking, this assumption is often too restrictive. Indeed, all possible associations cannot be identified a priori since targets can appear/disappear over time-hence the suggestion of two other frameworks: the "open world" [12] and the "extended open world" [19]. In the open world, $\Theta$ is exclusive but not exhaustive, so that a mass $m_{i}^{\Theta}(\emptyset)>0$ is possible. In this case, $\emptyset$ represents a reject class that describes the unknown hypotheses not taken into account during the problem formalization. The nonexhaustivity of the discernment frame can be managed by adding to $\Theta$ an alternative hypothesis $\{*\}$ representing all unknown propositions not explicitly defined in $\Theta$ [19]. This singleton allows the new discernment frame $\Theta_{\text {eow }}$ to become exhaustive [cf. (4)]. A nonzero value of $m_{i}^{\Theta_{\text {eow }}}(\emptyset)$ is then only linked to the sources' unreliability or to their discordance, i.e.,

$$
\Theta_{\mathrm{eow}}=\Theta \cup\{*\}
$$

The combination gathers the different sources bbm. In addition to all the existing operators [20], the conjunctive 


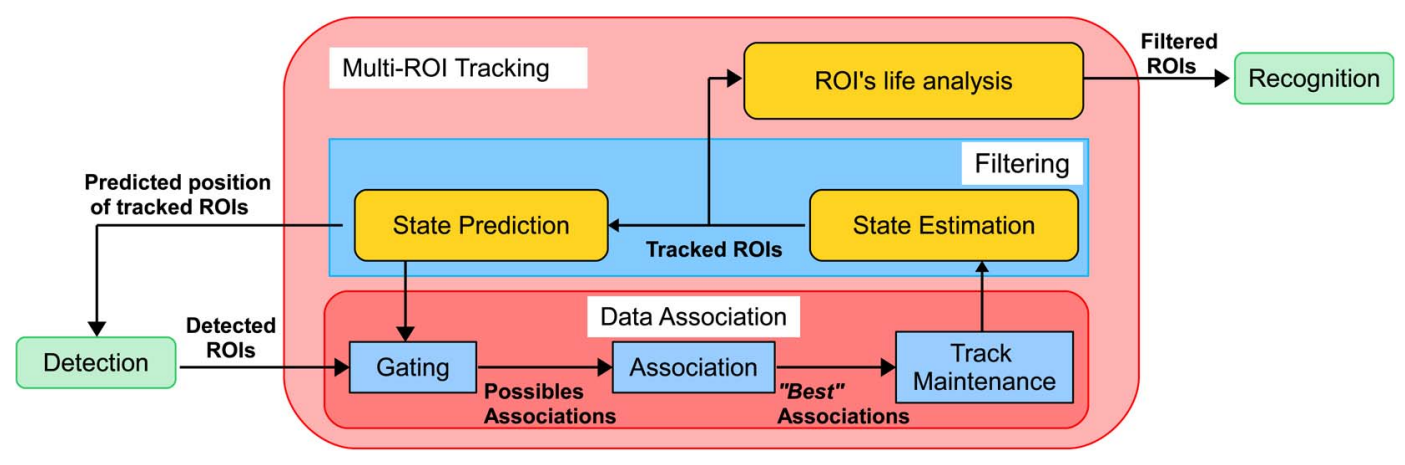

Fig. 6. Block diagram of the MRT algorithm.

combination rule is the most straightforward [27]. For $p$ sources to be combined, it is expressed as

$$
\left\{\begin{array}{lc}
m_{\cap}^{\Theta}(A)= & \sum_{A_{1} \cap \ldots \cap A_{p}=A} \prod_{j=1}^{p} m_{j}^{\Theta}\left(A_{j}\right), \\
m_{\cap}^{\Theta}(\emptyset)= & \sum_{A_{1} \cap \ldots \cap A_{p}=\emptyset} \prod_{j=1}^{p} m_{j}^{\Theta}\left(A_{j}\right) .
\end{array}\right.
$$

2) Pignistic Level: Finding the association relations consists in making a decision among all the possible hypotheses of the problem given. Usually, the decision is made in $\Theta$, i.e., on simple hypotheses $H_{j}$. Nevertheless, after the combination [see (5)], masses are placed either on singleton hypotheses $(|A|=1)$ or on unions $(|A|>1)$. It appears that a transformation from $2^{\Theta}$ to $\Theta$ is necessary. Among all the transformations available, the pignistic one [28] is commonly used. It is built as a probabilistic rule and shares the mass of $A$ on its singletons. The pignistic probability $($ BetP $)$ is given by

$$
\operatorname{BetP}\left(H_{j}\right)=\sum_{\substack{A \in 2^{\Theta} \\ H_{j} \in A}} \frac{m_{\cap}^{\Theta}(A)}{|A|\left(1-m_{\cap}^{\Theta}(\emptyset)\right)} \quad \text { with } m_{\cap}^{\Theta}(\emptyset)<1 \text {. }
$$

\section{B. Object Association: Problem Formalization}

In tracking algorithms using the TBM, the association task is observed from two different points of view [18]: target-to-track and track-to-target associations. This leads to the definition of two extended open worlds $\Theta_{i, .}$ and $\Theta_{., j},{ }^{4} i=1, \ldots, n$, with $n$ as the number of targets, and $j=1, \ldots, m$, with $m$ as the

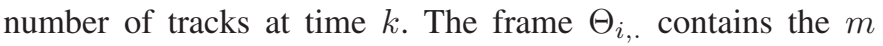
possible target $(i)$-to-track $(j)$ associations denoted $\left\{Y_{(i, j)}\right\}$, and $\left\{Y_{(i, *)}\right\}$ represents the appearance of $\operatorname{target}(i) .{ }^{5}$ The frame $\Theta_{., j}$ is composed of the $n$ possible track $(j)$-to-target $(i)$ assignments denoted $\left\{X_{(j, i)}\right\}$, and $\left\{X_{(j, *)}\right\}$ represents a track deletion, i.e.,

$$
\begin{aligned}
& \Theta_{i, .}=\left\{\left\{Y_{(i, 1)}\right\},\left\{Y_{(i, 2)}\right\}, \ldots,\left\{Y_{(i, m)}\right\},\left\{Y_{(i, *)}\right\}\right\} \\
& \Theta_{., j}=\left\{\left\{X_{(j, 1)}\right\},\left\{X_{(j, 2)}\right\}, \ldots,\left\{X_{(j, n)}\right\},\left\{X_{(j, *)}\right\}\right\}
\end{aligned}
$$

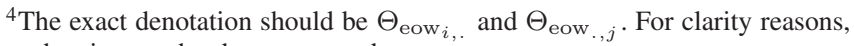
the subscript eow has been removed.

${ }^{5}$ The association of a target with the hypothesis $\{*\}$ means that no track is assigned to the target considered.
}

The question that is then raised is: "Is target $X_{i}$ associated with track $Y_{j}$ ?". To this question, three answers are possible: either they are associated or not, or the assignment is unclear. Within the TBM, these solutions will be described by three belief masses over $\left\{Y_{(i, j)}\right\}$, over its contrary $\overline{\left\{Y_{(i, j)}\right\}}$, and over the ignorance $\Theta_{i, \text {. }}$ so that

- $m_{j}^{\Theta_{i, \cdot}}\left(\left\{Y_{(i, j)}\right\}\right)$ : belief in " $X_{i}$ is associated with $Y_{j}$ ";

- $m_{j}^{\Theta_{i, .}}\left(\overline{\left\{Y_{(i, j)}\right\}}\right)$ : belief in " $X_{i}$ is not associated with $Y_{j}$ ";

- $m_{j}^{\Theta_{i, .}}\left(\Theta_{i, .}\right)$ : ignorance about the association.

The belief masses $m_{i}^{\Theta,, j}\left(\left\{X_{(j, i)}\right\}\right), m_{i}^{\Theta,, j}\left(\overline{\left\{X_{(j, i)}\right\}}\right)$, and $m_{i}^{\Theta}{ }^{\Theta}, j\left(\Theta_{., j}\right)$ are generated for the track-to-target associations in the same way. It is worth noting that no information is initially considered on $\left\{Y_{(i, *)}\right\}$ and $\left\{X_{(j, *)}\right\}$. These masses appear during combination. This finally leads to an $n \times m$ dimension assignment problem to be solved at each time step in order to define the association relations. The bbm's are then combined with the conjunctive rule (5) over each discernment frame $\Theta_{i, .}$ and $\Theta_{., j}$, generating masses $m^{\Theta_{i, .}}$ (and $m^{\Theta ., j}$ ) over $2^{\Theta_{i, .}}$ and $2^{\Theta_{., j}}[19]$, i.e.,

$$
\begin{gathered}
m^{\Theta_{i, .}}\left(\left\{Y_{(i, j)}\right\}\right)=m_{j}^{\Theta_{i, .}}\left(\left\{Y_{(i, j)}\right\}\right) \prod_{\substack{a=1 \\
a \neq j}}^{m} \alpha_{(i, a)} \\
m^{\Theta_{i, .}}\left(\left\{Y_{(i, j)}, Y_{(i, l)}\right\}\right)=m_{j}^{\Theta_{i, .}}\left(\Theta_{i, .}\right) m_{l}^{\Theta_{i, .}}\left(\Theta_{i, .}\right) \prod_{\substack{a=1 \\
a \neq j \\
a \neq l}}^{m} \beta_{(i, a)}
\end{gathered}
$$

and for union combinations of 2 to $m-1$ hypotheses, i.e.,

$$
m^{\Theta_{i, .}}\left(\left\{Y_{(i, j)}, \ldots, Y_{(i, l)}\right\}\right)=\gamma_{(i,(j, \ldots, l))} \prod_{\substack{a=1 \\ a \neq j \\ a \neq l}}^{m} \beta_{(i, a)}
$$

$m^{\Theta_{i, .}}(\{*\})=\prod_{a=1}^{m} \beta_{(i, a)}$

$m^{\Theta_{i, .}}\left(\overline{\left\{Y_{(i, j)}\right\}}\right)=m_{j}^{\Theta_{i, .}}\left(\overline{\left\{Y_{(i, j)}\right\}}\right) \prod_{\substack{a=1 \\ a \neq j}}^{m} m_{a}^{\Theta_{i, .}}\left(\Theta_{i, .}\right)$ 
TABLE I

Target-to-Track Pignistic Probabilities

\begin{tabular}{|c|c|c|c|c|c|}
\hline $\operatorname{BetP} P_{i, .}()$. & $Y_{1}$ & $\ldots$ & $Y_{m}$ & $*$ & $\emptyset$ \\
\hline$X_{1}$ & $\operatorname{Bet} P_{1, .}\left(Y_{(1,1)}\right)$ & $\ldots$ & $\operatorname{Bet} P_{1, .}\left(Y_{(1, m)}\right)$ & $\operatorname{Bet} P_{1, .}(*)$ & $\operatorname{Bet} P_{1, .}(\emptyset)$ \\
\hline$X_{2}$ & $\operatorname{Bet} P_{2, .}\left(Y_{(2,1)}\right)$ & $\ldots$ & $\operatorname{Bet} P_{2, .}\left(Y_{(2, m)}\right)$ & $\operatorname{Bet} P_{2, .}(*)$ & $\operatorname{Bet} P_{2, .}(\emptyset)$ \\
\hline$\vdots$ & $\vdots$ & $\vdots$ & $\vdots$ & $\vdots$ & $\vdots$ \\
\hline$X_{n}$ & $\operatorname{Bet} P_{n, .}\left(Y_{(n, 1)}\right)$ & $\ldots$ & $\operatorname{Bet} P_{n, .}\left(Y_{(n, m)}\right)$ & $\operatorname{Bet} P_{n, .}(*)$ & $\operatorname{Bet} P_{n, .}(\emptyset)$ \\
\hline
\end{tabular}

$m^{\Theta_{i, .}}\left(\Theta_{i, .}\right)=\prod_{a=1}^{m} m_{a}^{\Theta_{i, .}}\left(\Theta_{i, .}\right)$

$m^{\Theta_{i, .}}(\emptyset)=1-\left[\prod_{a=1}^{m} \alpha_{(i, a)}+\sum_{a=1}^{m} m_{a}^{\Theta_{i, .}}\left(\left\{Y_{(i, a)}\right\}\right) \prod_{\substack{b=1 \\ b \neq a}}^{m} \delta_{(i, b)}\right]$

with

$$
\left\{\begin{array}{l}
\alpha_{(i, a)}=\left(1-m_{a}^{\Theta_{i, .}}\left(\left\{Y_{(i, a)}\right\}\right)\right) \\
\beta_{(i, a)}=\left(m_{a}^{\Theta_{i, .}}\left(\overline{\left\{Y_{(i, a)}\right\}}\right)\right) \\
\gamma_{(i,(j, \ldots, l))}=m_{j}^{\Theta_{i, .}}\left(\Theta_{i, .}\right) \ldots m_{l}^{\Theta_{i, .}}\left(\Theta_{i, .}\right) \\
\delta_{(i, b)}=\left(1-m_{b}^{\Theta_{i, .}}\left(\left\{Y_{(i, b)}\right\}\right)\right) .
\end{array}\right.
$$

In order to make a decision, the pignistic transformation (6) is performed over the masses from (8). This leads to two pignistic matrices $\operatorname{Bet} P_{i, .}\left(\left\{Y_{(i, j)}\right\}\right)$ and $\operatorname{Bet} P_{., j}\left(\left\{X_{(j, i)}\right\}\right)$, as shown in Table I, for the target-to-track association. Each line defines the association probabilities of $X_{i}$ with $Y_{1}, \ldots, Y_{m}, *$. The association of a target $X_{i}$ or a track $Y_{j}$ with $*$ defines, respectively, the appearance/disappearance of a track. The column of the empty set $\emptyset$ defines the conflict in the target or track association considered. Usually, the conflict $m^{\Theta_{i, .}}(\emptyset)$ is redistributed through the normalization of the pignistic probability [see (6)]. Nevertheless, as in other studies [13], [17], [29], the authors use a nonnormalized form, i.e.,

$$
\begin{cases}\operatorname{Bet}_{i, .}\left(\left\{Y_{(i, j)}\right\}\right) & \triangleq \sum_{\substack{A \in \Theta_{i, .} \\ Y_{(i, j)} \in A}} \frac{m^{\Theta_{i, .}(A)}}{|A|} \\ \operatorname{Bet}_{i, .}(\emptyset) & \triangleq m^{\Theta_{i, .}(\emptyset) .}\end{cases}
$$

Because of its informative aspect for decision making [20], the conflict is conserved, particularly for the detection of association ambiguities or contradictions. The formalization of (9) to the multiobject association problem can be found in [17]. Decision making is based on these pignistic matrices. In the literature, several algorithms are proposed for selecting the "best" associations regarding global decision cost functions [13]. Generally, and it will be the case in this work, they consider three constraints [18].

- A target can only be associated with one track, and vice versa.

- Multiple existing tracks can disappear.

- Multiple new tracks can appear.

\section{Multi-ROI Association And Filtering}

\section{A. Track Filtering}

Tracking a moving target can be viewed as the observation of a dynamic system. That is why the straightforward solution employed in computer-vision tracking applications is the KF [22]. Usually, a tracking filter is derived in terms of a recursive (linear) estimator. It recursively computes the estimates of the object $l$-dimensional state vector $x_{j}(k) \in \mathbb{R}^{l}$ by a combination of the previous estimates and new object observations $z_{i}(k) \in \mathbb{R}^{p}$. For this purpose, a dynamic (motion) model and a measurement model are employed, i.e.,

$$
\begin{aligned}
x_{j}(k) & =F x_{j}(k-1)+w(k), \\
z_{i}(k) & =H x_{j}(k)+v(k) .
\end{aligned}
$$

$F$ is the $l \times l$ state transition matrix based on the chosen track motion model. It is assumed that the model and measurement noises $w$ and $v$ are independent and identically distributed random noises. The state noise $w$ is a white Gaussian $l$-dimensional process with zero mean and covariance $Q$ denoted $w \sim \mathcal{N}_{l}(0, Q)$ and $v \sim \mathcal{N}_{p}(0, R)$. The measurement model relates the state $x_{j}(k)$ to the observation $z_{i}(k)$ with the measurement matrix $H \in \mathbb{R}^{p \times l}$. For a given frame $k$, the detector provides a set $\mathcal{Z}$ of ROI candidates, which represents the $n$ targets defined by the measurements $z_{i}(k) \in \mathcal{Z}$. Assume that the state evolution model linearly behaves with respect to the track motion and that the observations $z_{i}(k)$ and states $x_{j}(k)$ are linearly dependent, leading to (10). According to the data association results at time $k$, KFs corresponding to existing tracks are updated, and new filters are created for appearing tracks. The number $m$ of KFs depends on the number of tracked ROIs.

The 2-D motion of objects in a video sequence is strongly related to the camera motion and to their own displacement. In TSR applications, only the camera motion has to be considered since the objects are static. A steady-state vehicle movement is assumed, characterized by low velocity variations with respect to the sampling rate of the system ( $>15 \mathrm{fps})$. Moreover, the angle between the line of sight of the camera and the vehicle direction is sufficiently small, so that the sign size $s$ in the image can be considered as constantly increasing. In this context, the nearly constant velocity model (12) represents a globally satisfactory motion model and a classic choice for tracking applications [3], [8], [30]. The state vector $x_{j}(k)$ of a $\operatorname{track}(j)$ is such that

$$
x_{j}(k)=\left[x, y, s, v_{x}, v_{y}, v_{s}\right]^{T}
$$

where $[x, y]$ defines the position, $s$ is the size of the tracked ROI $j$ in the image coordinate frame, $\left[v_{x}, v_{y}\right]$ is the $\operatorname{track}(j)$ relative velocities between two successive frames, and $v_{s}$ is the scale change. Due to the high sampling time of the MRT, the scale variation $v_{s}(k)$ and the position variations $\left[v_{x}(k), v_{y}(k)\right]$ of the 
traffic signs are considered similar to the previous ones and only due to Gaussian noises, i.e.,

$$
\begin{gathered}
\left\{\begin{array}{l}
x(k)=x(k-1)+v_{x}(k), \\
y(k)=y(k-1)+v_{y}(k), \\
s(k)=s(k-1)+v_{s}(k) .
\end{array}\right. \\
\text { with : }\left\{\begin{array}{l}
v_{x}(k) \sim \mathcal{N}\left(v_{x}(k-1), \sigma_{x}^{2}\right), \\
v_{y}(k) \sim \mathcal{N}\left(v_{y}(k-1), \sigma_{y}^{2}\right), \\
v_{s}(k) \sim \mathcal{N}\left(v_{s}(k-1), \sigma_{s}^{2}\right)
\end{array}\right.
\end{gathered}
$$

$z_{i}(k)$ is a $p \times 1$ vector defined by

$$
z_{i}(k)=[x, y, s]^{T} .
$$

Three processing steps are possible for a given track: creation, update, and deletion. The appearance decision (depending on $\left.\operatorname{Bet} P_{i, .}\left(\left\{Y_{(i, *)}\right\}\right)\right)$ creates a new filter $j$. Its state vector $x_{j}(k)$ is initialized by the observation $z_{i}(k)$ of the newly detected ROIs. The initial displacement and scale change are set to 0 . An association between an existing track and a target leads to the track update. In addition, the update step helps to increment a count $c_{\text {update }}(j)$ related to $\operatorname{track}(j)$, which will be used to remove the false positives. The disappearance decision (related to $\left.\operatorname{Bet} P_{., j}\left(\left\{X_{(j, *)}\right\}\right)\right)$ follows a non-association between the targets and the track considered. The track predicted state $\hat{x}_{j}(k)$ is propagated into filter $j$ with no correction (15). A track deletion occurs following three consecutive disappearances. $\hat{x}_{j}(k)$ is fed back to Detection as a priori search positions of the given signs in the coming images in order to reduce the ROI search space and time, i.e.,

$$
\begin{aligned}
& \hat{x}_{j}(k)=F x_{j}(k-1) \\
& \hat{P}_{j}(k)=F P_{j}(k-1) F^{T}+Q .
\end{aligned}
$$

$\hat{P}_{j}(k)$ defines the predicted error covariance matrix of filter $j$, and $P_{j}(k-1)$ defines its error covariance matrix in frame $k-$ 1. The false positive reduction is performed through a trackrelated confidence score $C(j)$ defined by the ratio between the update count $c_{\text {update }}(j)$ and the length of the track, i.e.,

$$
C(j)=\frac{c_{\text {update }}(j)}{\text { length }(j)}
$$

with $c_{\text {update }}(j)$ as the number of frames where ROI $j$ is confirmed, and length $(j)$ as the total frame number where track $j$ is present. A false positive occurs when $C(j)$ is lower than a predefined threshold $T_{\text {confidence; }}$ otherwise, the track $j$ is valid and becomes an ROI forwarded to Recognition.

\section{B. Data Modeling in the TBM}

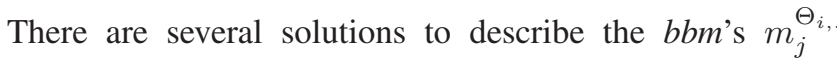
and $m_{i}^{\Theta}{ }^{\Theta, j}$ related to $\left\{Y_{(i, j)}\right\}$ and $\left\{X_{(j, i)}\right\}$. One model considers, for instance, nonantagonistic propositions in which $m_{j}^{\Theta_{i, .}}\left(\left\{Y_{(i, j)}\right\}\right)$ and $m_{j}^{\Theta_{i, \cdot}}\left(\overline{\left\{Y_{(i, j)}\right\}}\right)$ cannot simultaneously be nonzero in order to avoid intrinsic conflict [13], [18], [20]. In this paper, the masses defined, respectively, on $\Theta_{i, .}$ and $\Theta_{., j}$ are described according to [31]

$$
\left\{\begin{array}{l}
m_{j}^{\Theta_{i, .}}\left(\left\{Y_{(i, j)}\right\}\right)=\alpha_{j} \exp ^{-d_{i j}^{2}}, \\
m_{j}^{\Theta_{i, .}}\left(\overline{\left\{Y_{(i, j)}\right\}}\right)=\alpha_{j}\left(1-\exp ^{-d_{i j}^{2}}\right), \\
m_{j}^{\Theta_{i, .}}\left(\Theta_{i, .}\right)=1-\alpha_{j}
\end{array}\right.
$$

where $0<\alpha_{j}<1$ represents the reliability of source $j$, and $d_{i j}$ is the dissimilarity measurement between target $(i)$ detected at time $k$ and $\operatorname{track}(j)$ already known at time $k$. $d_{i j}$ characterizes the level of confidence that target $(i)$ corresponds to $\operatorname{track}(j)$. For the purpose of ROI tracking, $d_{i j}$ is chosen as the Mahalanobis distance [7] between $\operatorname{track}(j)$ and $\operatorname{target}(i)$, i.e.,

$$
d_{i j}^{2}=\tilde{z}_{i j}^{T} S^{-1} \tilde{z}_{i j}
$$

with $S$ as the residual covariance matrix, and $\tilde{z}_{i j}$ as the innovation of filter $j$. In (17), the choice of a monotone decreasing function of $d_{i j}$ leads to the belief reduction in the association of $\operatorname{target}(i)$ to $\operatorname{track}(j)$ when the distance between the objects is large. On the contrary, if the dissimilarity measurement is low, their association is more probable. It can be noted that $d_{i j}$ depends on the tracking filter covariance, so that the belief depends on the prediction and tracking performance.

\section{Pignistic Association Algorithm}

In [17], a review of the state-of-the-art credal association algorithms is proposed. It shows that with a global (suboptimal) cost optimization, suspicious associations could be selected. To cope with this problem, local optimization solutions have been proposed. In the MRT, decision making is based on the local pignistic probability (LPP) algorithm (cf. Algorithm 1) [17]. This algorithm is applied to each pignistic matrix $\operatorname{Bet}_{i, .}\left(\operatorname{Bet}_{., j}\right)$ and performs a successive linewise selection of the $n$ (or $m$ ) local maximum in the pignistic matrix. Compared to other algorithms, the LPP has the advantage of generating decisions directly from the pignistic matrices with no extra calculations. A major consequence is its ability to be used in real-time applications. Furthermore, the LPP is less conservative than other association solutions. A complete description and evaluation of this algorithm can be found in [17].

Algorithm 1 Local Pignistic Probability (LPP $(\operatorname{Bet} P))$

Require: Pignistic matrix $\operatorname{Bet} P$

Ensure: Decision vector containing the "best" associations $\{$ Associations $\} \leftarrow\{\}$

for all lines of $\operatorname{Bet} P$ do

$\left(i_{\max }, j_{\max }\right)=\arg \max _{(i, j)}(\operatorname{Bet} P(i, j))$

$\{$ Associations $\} \leftarrow\left(i_{\max }, j_{\max }\right)$

Remove $i_{\max }$ row and $j_{\max }$ column from $\operatorname{Bet} P$

end for 


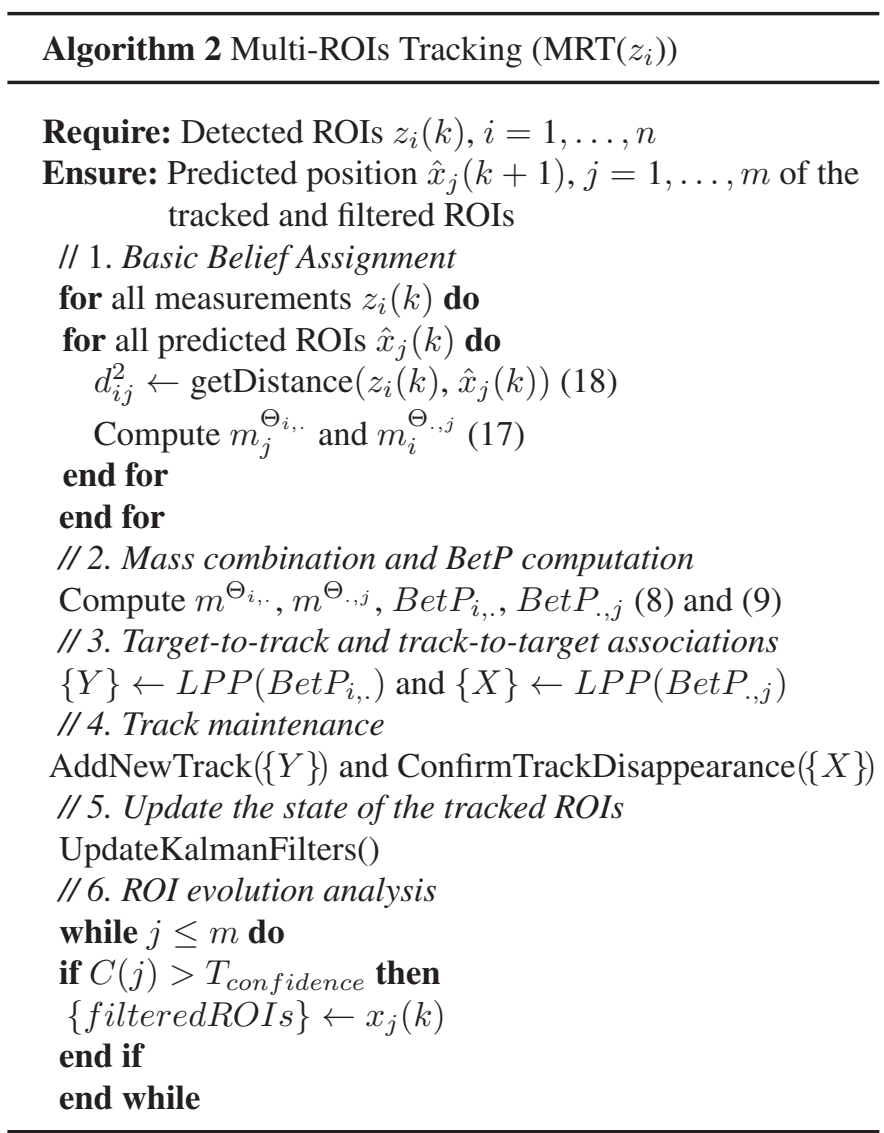

An overview of the MRT algorithm is shown in Algorithm 2. After the basic belief assignment and the bbm's combination (steps 1 and 2), the best target-to-track and track-to-target associations are generated by the LPP (step 3). Track maintenance (step 4) manages track appearance and disappearance. The update step of the tracking filters is performed in step 5 depending on the assignment decisions and available observations $z_{i}(k)$. To deal with false positives, the evolution of the tracked ROIs is analyzed (step 6).

\section{EXPERIMENTAL RESULTS}

This section presents the results obtained during real-time experiments performed with a fully equipped test vehicle. First, the configuration of the system and implementation details are presented; then, a quantitative analysis presents the performance rates of the MRT. In order to give a realistic idea of the system performance in real conditions, a video (video_IEEE.wmv) is also provided.

\section{A. System Configuration}

The vehicle-mounted camera is a 12-bit gray-scale ECK100 from Sensata Technologies providing VGA images $(640 \times$ 480 pixels, $25 \mathrm{fps}$ ) of the vehicle front scene. The MRT system is written in C++ with the OpenCV 2.4 library. It runs on an Intel core i7 $2.20 \mathrm{GHz}$ with $8 \mathrm{~GB}$ RAM under Windows 7 (64 bits). The critical parameters of the application are as follows: $T_{\text {confidence }}=0.85$ (confidence threshold of the track evolution rate $C(j)), \alpha_{j}=0.9$ (discounting factor of the bbm), $m_{\max }=10$ (maximal number of KFs), $\left(\sigma_{x}, \sigma_{y}, \sigma_{s}\right)=$
TABLE II

TEST SEQUENCES ON FRENCH ROADS

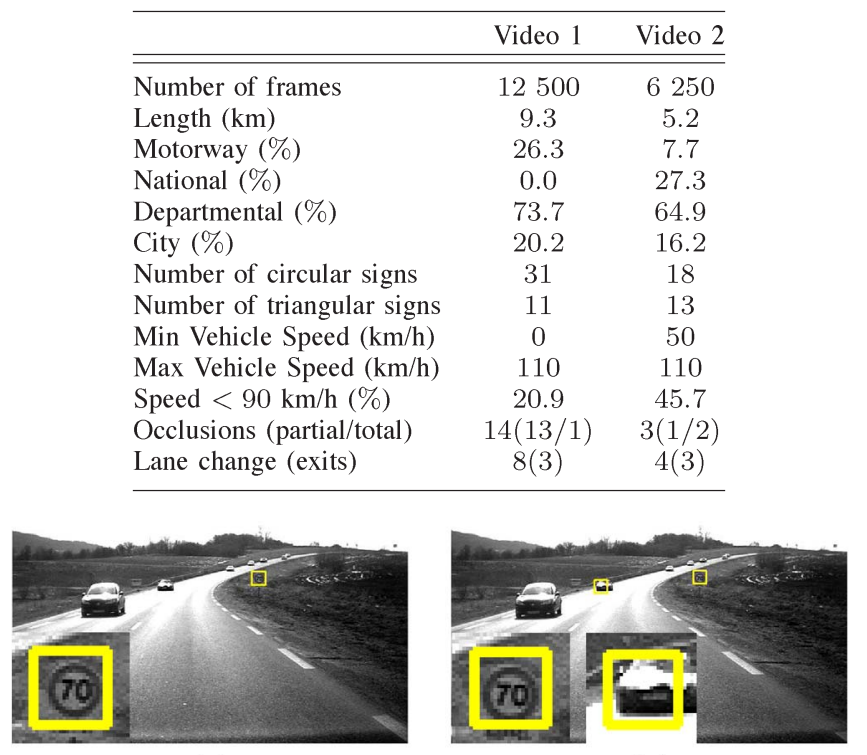

(a)

(b)

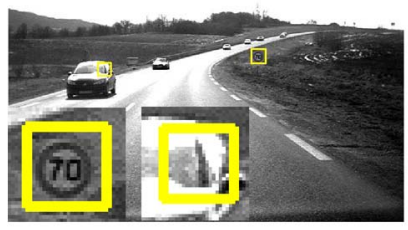

(c)

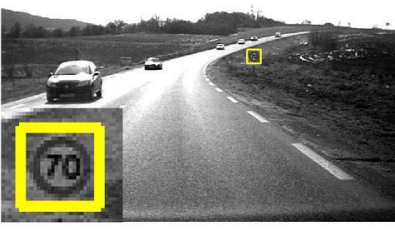

(d)
Fig. 7. Detection results without MRT in successive frames. (a) and (d) Correct sign detection. (b) and (c) Presence of false positives.

$(2,2,3)$ (standard deviations of the Kalman motion model), and the minimum size of an ROI to be detected is $20 \times$ 20 pixels. The performance of the MRT refers to its capacity to reduce the false positives while maintaining a high detection rate. Consequently, this section focuses on the detection rate per frame (DRPF), the false positive per frame (FPPF), and the detection rate per sign (DRPS) for system evaluation. A system output is defined as a true positive (TP) if it can be matched with the ground truth and as a false positive (FP) if otherwise. A tracked target is correct if a sign candidate is forwarded to the recognition step more than $N=3$ successive times. The DRPF, DRPS, and FPPF are defined as

$$
\begin{aligned}
& \text { DRPF }=\frac{\text { Number of true positives }}{\text { Ground Truth }} \cdot 100 \\
& \text { DRPS }=\frac{\text { Number of correct detections }}{\text { Total Sign Number }} \cdot 100 \\
& \text { FPPF }=\frac{\text { Number of false positives }}{\text { Total Frame Number }} .
\end{aligned}
$$

\section{B. Quantitative Results}

The MRT performance is evaluated with respect to the ground truth of the two video sequences described in Table II. These sequences have been annotated empirically so that, in each frame, the positions of signs having between $20 \times$ 20 pixels and $60 \times 60$ pixels are defined. The first sequence 


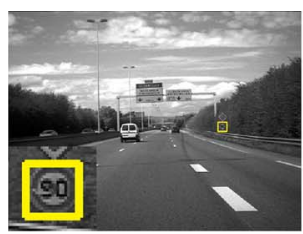

(a)

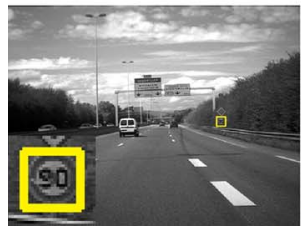

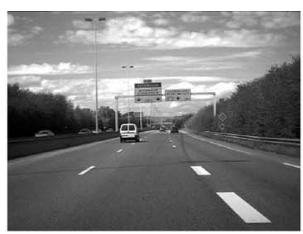

(b)

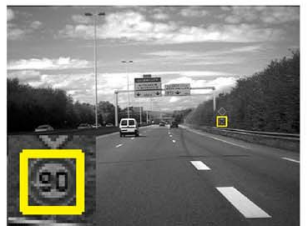

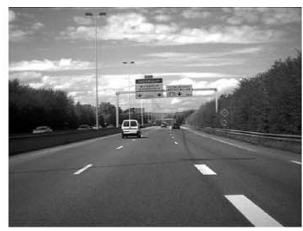

(c)

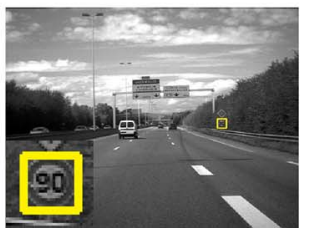

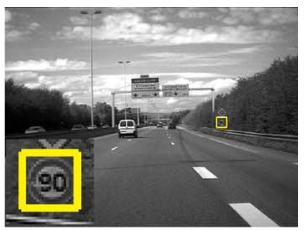

(d)

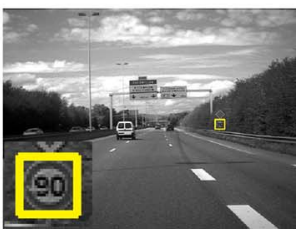

Fig. 8. Impact of the candidate position prediction feedback in four successive frames. (Top) Without feedback. (Bottom) With feedback.

TABLE III

Comparison Between MRT and State-OF-THE-ARt Detection-Tracking-Recognition Algorithms

\begin{tabular}{lllllll}
\hline Paper & $\begin{array}{l}\text { Evaluation data } \\
\text { format }\end{array}$ & $\begin{array}{l}\text { Number of } \\
\text { positive ROIs }\end{array}$ & $\begin{array}{l}\text { Best detection } \\
\text { rate (DRPF)(\%) }\end{array}$ & $\begin{array}{l}\text { False positives } \\
\text { for best DRPF }\end{array}$ & Mean DRPF & $\begin{array}{l}\text { Mean false } \\
\text { positives }\end{array}$ \\
\hline \hline$[32]$ & 2134 images from videos & 3298 & 94.03 & FPPF: 3.41 & - & - \\
{$[33]$} & 2967 images from videos & 4886 & 90.1 & PPV: $85.6 \%$ & - & - \\
{$[34]$} & Images from 34 videos & N/A & 87.12 & FPR: $0.14 \%$ & - & - \\
Our paper & 18000 images from two videos & 2850 & 89.92 & FPPF: 0.16 & 74.19 & FPPF: 0.13 \\
\hline
\end{tabular}

TABLE IV

Properties of Selected Detection-Tracking-Recognition Algorithms

\begin{tabular}{llllll}
\hline Paper & Sign type detected & Real-time & Rotation inv. & Model vs. training & Test image type \\
\hline \hline$[32]$ & Circular red and blue & Yes & Yes & Both & Low-res \\
{$[33]$} & Circular red & No & Yes & Training & Low-res \\
{$[34]$} & Circular & Yes & Yes & Model & Low-res \\
Our paper & Circular, triangular, and yield signs & Yes & Yes & Model & Low-res \\
\hline
\end{tabular}

(12500 frames) mainly concerns departmental roads and motorways with several lane changes and thus presents large vehicle speed variations. Forty-two traffic signs appear in 1452 frames. The second video (6250 frames) contains even more various scenarios since all French road types are represented with a larger ratio of speeds under $90 \mathrm{~km} / \mathrm{h}$. This variety is confirmed by the high ratio of signs with respect to its length. These signs are present in 1031 frames.

Fig. 7 gives a qualitative view of detection results without MRT on consecutive frames. In frames (b) and (c), two false positives can be observed. As they occur suddenly and do not remain visible over time, these are typical examples of wrong detections that can be removed by the MRT. Fig. 8 (top) shows a short sequence of circular sign detection with no feedback between Tracking and Detection. In frame (a), the sign is correctly detected. However, in frames (b) and (c), the corner detection fails and thus misses the circular sign twice. This drawback is solved by the prediction task of the MRT and the feedback of the tracked ROIs (see Fig. 8, bottom), providing the detector with potential search positions.

A comparison of the proposed MRT with DetectionTracking-Recognition approaches from [32]-[34] and evaluated in [1] is proposed in Table III. Some properties of these systems are given in Table IV as they are presented in [1]. One may note that a direct metric comparison is inappropriate since each method is evaluated considering specific data sets and sometimes different metrics. For instance, in [33] and [34], two measurements are used to evaluate the FP: the positive predictive value $(\mathrm{PPV}=\mathrm{TP} /(\mathrm{TP}+\mathrm{FP}))$ and the false positive rate $(\mathrm{FPR}=\mathrm{FP} / N$, where $N$ is the number of negative examples in the data set). Unlike the FPPF, the PPV is influenced by the number of frames where signs are present. Consequently, it is difficult to compare the proposed method with [34] as the authors do not give any information about the 34 sequences used. Moreover, the FPR is rarely used as the negative number does not make sense [1]. Considering these limitations, the proposed solution presents interesting performance with a low FPPF (0.16) and a high DRPF (89.92). The mean DRPF and mean false positives (more accurate since they show the results for different sign types) already highlight the good results of the MRT.

Table $\mathrm{V}$ shows that the circular and triangular sign detector alone (denoted $\overline{M R T}$ ) provides, respectively, $87 \%$ and $91 \%$ of DRPS for Video 1 with 0.28 and 0.26 of FPPF. Slightly better results are obtained in Video 2. In addition, it is clearly visible that the MRT reduces the false positives by more than $45 \%$ while keeping substantially similar levels of DRPF and DRPS. This lowers the amount of useless data provided to the recognition step and thus reduces the global TSR computational time. Fig. 9 presents more details about the MRT influence on the detection step. These receiver operating characteristic (ROC) curves are created by plotting the DRPF with respect 
TABLE V

PERFORMANCE RATES FOR VIDEO 1 AND 2

\begin{tabular}{|c|c|c|c|c|}
\hline & \multicolumn{2}{|l|}{ Video 1} & \multicolumn{2}{|c|}{ Video 2} \\
\hline & $\overline{M R T}$ & $M R T$ & $\overline{M R T}$ & $M R T$ \\
\hline \multicolumn{5}{|l|}{ Circular sign } \\
\hline DRPS $(\%)$ & 87 & 87 & 95 & 95 \\
\hline DRPF $(\%)$ & 87.95 & $86.55(-2 \%)$ & 92.81 & $93.3(+0.5 \%)$ \\
\hline$F P P F$ & 0.28 & $0.15(-46 \%)$ & 0.27 & $0.17(-37 \%)$ \\
\hline \multicolumn{5}{|l|}{ Triangular sign } \\
\hline DRPS (\%) & 91 & 91 & 100 & 100 \\
\hline$D R P F(\%)$ & 64.15 & $55.37(-14 \%)$ & 69.54 & $61.56(-11 \%)$ \\
\hline$F P P F$ & 0.26 & $0.14(-46 \%)$ & 0.11 & $0.07(-37 \%)$ \\
\hline
\end{tabular}

to the FPPF, considering various system configurations with and without the MRT. For the circular sign detection, the ROC curves clearly show the improvement induced by the MRT algorithm since equivalent DRPFs are obtained with lower FPPF values [see Fig. 9(a)], particularly when the DRPF is high $(>80 \%)$. For the triangular sign detection, the reduction of FPPF is also large (about 40\%) but with a lower DRPF (71\%) than for circular signs (87\%), as shown in Fig. 9(b). In this second case, the test highlights the sensitivity of the triangular detector to the minimum ROI size. Improved results can be obtained by considering ROIs larger than 30 square pixels.

Table VI shows the average computation time per frame in milliseconds, without code optimization, for the detector and the MRT in both sequences. The detection of triangular and circular signs requires an average of $40 \mathrm{~ms}$ to process a single frame. The MRT computation time depends on the number of detected $n$ and tracked $m$ ROIs but remains insignificant (less than $2 \mathrm{~ms}$ in the worst case). With $n=m=4$, the computation time of the MRT is close to $0.70 \mathrm{~ms}$, representing less than $2 \%$ of the computation time of the detection. Therefore, the proposed MRT provides good performance by reducing false positives while run time remains low.

\section{CONCLUSiON}

A multi-ROI association and tracking algorithm based on spatiotemporal data fusion for vision-based TSR has been presented. The idea is to reduce false positive detections by tracking sign candidates over time and processing the association task with the TBM. First, a detector extracts a set of ROI candidates, which represents data to be combined in the fusion process. Then, the correlation task provides the associations with respect to the maximum of belief of each target-to-track and track-to-target pairing. Tracking is performed using multiple KFs. Finally, the generated tracks are analyzed to detect false positives. The algorithm provides a set of ROIs, and on the other hand, it feeds the predicted sign locations back into the detector as a priori knowledge to influence Detection in subsequent frames. The experimental results show the effectiveness of this strategy in the reduction of the false positives by up to $45 \%$ with real-time performance. It can be noted that the multiROI tracker does not only apply to the ROI detector used in this paper. It can be applied to any traffic sign detector to reduce false positives.

This paper has focused on tracking traffic signs leading to a limited number of ROIs in frames. One extension of this study

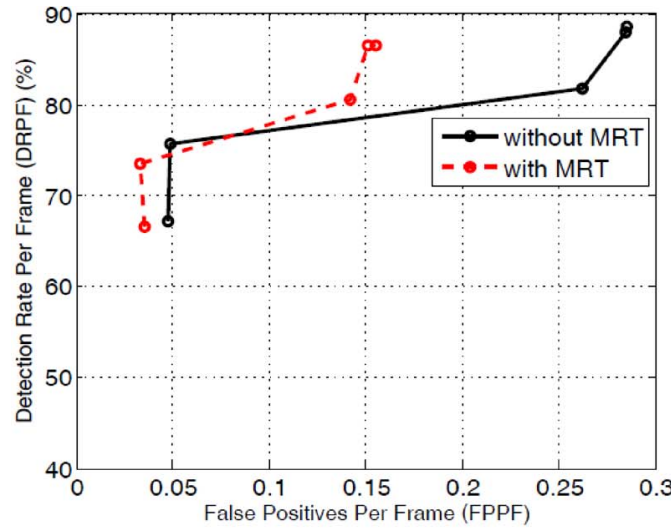

(a)

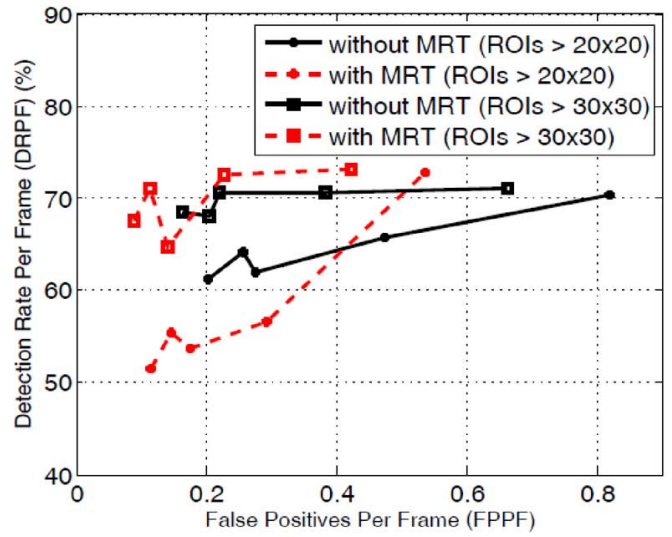

(b)

Fig. 9. ROC curves without and with MRT for (a) circular and (b) triangular signs.

TABLE VI

Average Computation Times

\begin{tabular}{ll}
\hline & Execution time $(\mathrm{ms})$ \\
\hline \hline Detection (triangular and circular) & 40 \\
$\operatorname{MRT}(n=1, m=1)$ & 0.25 \\
$\operatorname{MRT}(n=2, m=2)$ & 0.30 \\
$\operatorname{MRT}(n=3, m=3)$ & 0.56 \\
$\operatorname{MRT}(n=4, m=4)$ & 0.65 \\
$\operatorname{MRT}(n=5, m=5)$ & 1.56
\end{tabular}

will be to consider more dynamic situations in a dense traffic environment, such as pedestrian and dynamic object tracking.

\section{REFERENCES}

[1] A. Møgelmose, M. M. Trivedi, and T. B. Moeslund, "Vision-based traffic sign detection and analysis for intelligent driver assistance systems: Perspectives and survey," IEEE Trans. Intell. Transp. Syst., vol. 13, no. 4, pp. 1484-1497, Dec. 2012.

[2] N. Barnes, A. Zelinsky, and L. Fletcher, "Real-time speed sign detection using the radial symmetry detector," IEEE Trans. Intell. Transp. Syst., vol. 9, pp. 322-332, Jun. 2008.

[3] M. A. García-Garrido, M. A. Sotelo, and E. Martín-Gorostiza, "Fast traffic sign detection and recognition under changing lighting conditions," in Proc. IEEE ITSC, 2006, pp. 811-816.

[4] F. Zaklouta and B. Stanciulescu, "Warning traffic sign recognition using a HOG-based K-d tree," in Proc. IEEE IV Symp., 2011, pp. 1019-1024.

[5] J. Greenhalgh, M. Mirmehdi, and S. Member, "Real-time detection and recognition of road traffic signs," IEEE Trans. Intell. Transp. Syst., vol. 13, no. 4, pp. 1498-1506, Dec. 2012.

[6] Y. Bar-Shalom and X.-R. Li, Multitarget-Multisensor Tracking: Principles and Techniques. Brandford, U.K.: YBS Publishing, 1995. 
[7] S. Blackman and R. Popoli, Design and Analysis of Modern Tracking System. Norwood, MA, USA: Artech House, 1999.

[8] A. Ruta, Y. Li, and X. Liu, "Real-time traffic sign recognition from video by class-specific discriminative features," Pattern Recog., vol. 43, no. 1, pp. 416-430, Jan. 2010.

[9] D. B. Reid, "An algorithm for tracking multiple targets," IEEE Trans. Autom. Control, vol. 24, no. 6, pp. 843-854, Dec. 1979.

[10] A. Dempster, "Upper and lower probabilities induced by a multivalued mapping," Ann. Math. Statist., vol. 38, no. 2, pp. 325-339, 1967.

[11] G. Shafer, A Mathematical Theory of Evidence. Princeton, NJ, USA: Princeton Univ. Press, 1976.

[12] P. Smets and R. Kennes, "The transferable belief model," Artif. Intell., vol. 66, no. 2, pp. 191-234, Apr. 1994.

[13] B. Mourllion, D. Gruyer, C. Royère, and S. Théroude, "Multi-hypotheses tracking algorithm based on the belief theory," in Proc. IEEE Conf. Inf. Fusion, 2005, vol. 2.

[14] P. Smets and B. Ristic, "Kalman filter and joint tracking and classification based on belief functions in the TBM framework," Inf. Fusion, vol. 8, no. 1, pp. 16-27, Jan. 2007.

[15] B. Ristic and P. Smets, "The TBM global distance measure for the association of uncertain combat ID declarations," Inf. Fusion, vol. 7, no. 3, pp. 276-284, Sep. 2006.

[16] A. Ayoun and P. Smets, "Data association in multi-target detection using the transferable belief model," Int. J. Intell. Syst., vol. 16, no. 10, pp. 1167-1182, 2001.

[17] J. Daniel and J.-P. Lauffenburger, "Multi-object association decision algorithms with belief functions," in Proc. IEEE Conf. Inf. Fusion, 2012, pp. 669-676.

[18] M. Rombaut, "Decision in multi-obstacle matching process using the theory of belief," in Proc. IEEE Conf. AVCS, 1998, pp. 63-68.

[19] C. Royère, D. Gruyer, and V. Cherfaoui, "Data association with belief theory," in Proc. IEEE Conf. Inf. Fusion, 2000.

[20] J. Daniel and J.-P. Lauffenburger, "Fusing navigation and vision information with the transferable belief model: Application to an intelligent speed limit assistant," Inf. Fusion, vol. 18, pp. 62-77, Jul. 2014.

[21] M. Boumediene, C. Cudel, M. Basset, and A. Ouamri, "Triangular traffic signs detection based on RSLD algorithm," Mach. Vis. Appl., vol. 24, no. 8, pp. 1721-1732, Nov. 2013.

[22] R. E. Kalman, "A new approach to linear filtering and prediction problems," J. Basic Eng., vol. 82, no. 1, pp. 35-45, 1960.

[23] M. García-Garrido et al., "Robust traffic signs detection by means of vision and V2I communications," in Proc. IEEE ITSC, 2011, pp. 10031008.

[24] C.-Y. Fang, S.-W. Chen, and C.-S. Fuh, "Road-sign detection and tracking," IEEE Trans. Veh. Technol., vol. 52, no. 5, pp. 1329-1341, Sep. 2003

[25] S. Šegvić, K. Brkić, Z. Kalafatić, and A. Pinz, "Exploiting temporal and spatial constraints in traffic sign detection from a moving vehicle," Mach. Vis. Appl., vol. 25, no. 3, pp. 649-665, Apr. 2014.

[26] C. Harris and M. Stephens, "A combined corner and edge detector," in Proc. AVC, 1988, pp. 147-152.

[27] P. Smets, "Analyzing the combination of conflicting belief functions," Inf. Fusion, vol. 8, no. 4, pp. 387-412, Oct. 2007.

[28] P. Smets, "Constructing the pignistic probability function in a context of uncertainty," Uncertain. Artif. Intell., vol. 5, pp. 29-40, 1990.

[29] J.-P. Lauffenburger, J. Daniel, and O. Saif, "Multi-object association with belief functions: The dual decision algorithm," in Proc. IFAC ACATTA Workshop, 2013, pp. 193-198.

[30] C. G. Cifuentes, M. Sturzel, F. Jurie, and G. Brostow, "Motion models that only work sometimes," in Proc. Conf. BMV, 2012, pp. 55.1-55.12.

[31] T. Denœux, "A $k$-nearest neighbor classification rule based on DempsterShafer theory," IEEE Trans. Syst., Man, Cybern., vol. 25, no. 5, pp. 804813, May 1995.

[32] Y. Gu, T. Yendo, M. Panahpour Tehrani, T. Fujii, and M. Tanimoto, "Traffic sign detection in dual-focal active camera system," in Proc. IEEE IV Symp., 2011, pp. 1054-1059.

[33] D. Deguchi, M. Shirasuna, K. Doman, I. Ide, and H. Murase, "Intelligent traffic sign detector: Adaptive learning based on online gathering of training samples," in Proc. IEEE IV Symp., 2011, pp. 72-77.

[34] M. Meuter, C. Nunn, S. M. Görmer, S. Müller-Schneiders, and A. Kummert, "A decision fusion and reasoning module for a traffic sign recognition system," IEEE Trans. Intell. Transp. Syst., vol. 12, no. 4, pp. 1126-1134, Dec. 2011.

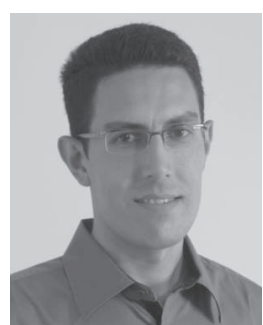

Mohammed Boumediene received the M.Eng. degree in computer science and the M.Sc. degree in image processing from the Université des Sciences et de la Technologie d'Oran Mohamed Boudiaf (USTOMB), Oran, Algeria, in 2004 and 2006, respectively. $\mathrm{He}$ is currently working toward the $\mathrm{Ph} . \mathrm{D}$. degree, with focus on traffic sign recognition systems, in the Laboratoire Signaux et Images, USTOMB. His research interests include computer vision, object detection, object association, and intelligent transportation systems.

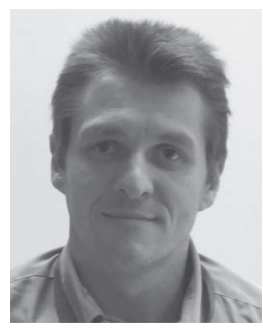

Jean-Philippe Lauffenburger received the M.Sc. and Ph.D. degrees in electrical and control engineering from the Université de Haute Alsace (UHA), Mulhouse, France, in 1998 and 2002, respectively.

Since 2004, he has been an Assistant Professor with UHA. His major research interests are data fusion, navigation, and joint association and tracking for intelligent transportation systems.

Dr. Lauffenburger is a member of the International Federation of Automatic Control (IFAC) "Transportation Systems" TC7.4 and a reviewer for the Information Fusion Journal. He is also a regular Associate Editor for IEEE and IFAC conferences.

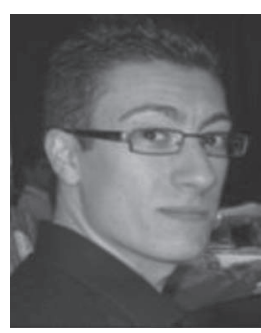

Jérémie Daniel received the M.Sc. and Ph.D. degrees in control theory and signal processing from the Université de Haute Alsace (UHA), Mulhouse, in 2007 and 2010, respectively.

Since 2010, he has been a Research and Algorithm Development Engineer with the UHA. His research interests include data fusion and reference generation for advanced driver assistance systems.

Dr. Daniel is a regular reviewer for the IEEE TRANSACTIONS ON INTELLIGENT TRANSPORTATION SYSTEMS.

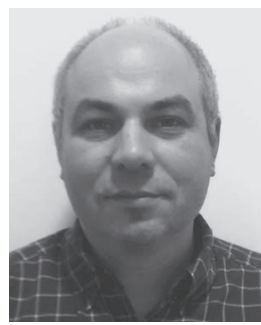

Christophe Cudel received the Ph.D. degree in automatic, signal, and image processing from the Université de Reims Champagne-Ardenne, Reims, France, in 1995.

After a one-year postdoctoral position in a computer vision company, he joined the Laboratoire Modélisation Intelligence Processus et Systèmes, Université de Haute Alsace, Mulhouse, France, where he is currently an Associate Professor. His main research topics are in computer vision, eye tracking, 3-D vision, and vision for driving assistance.

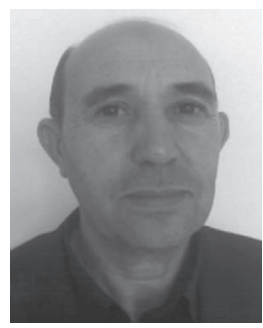

Abdelaziz Ouamri received the Engineer degree in electrical engineering from the École Nationale Supérieure d'Ingénieurs de Caen, Caen, France; and the M.Sc. degree in automatic and signal processing, the $\mathrm{Ph} . \mathrm{D}$. degree in engineering, and the "Doctorat d'Etat" in signal processing from the Universite Paris XI, Paris, France, in 1979, 1981, and 1986, respectively.

He is currently a Professor with the Université des Sciences et de la Technologie d'Oran Mohamed Boudiaf, Oran, Algeria. His research interests are focused on high-resolution spectral array processing methods and detection and tracking of moving objects.

Dr. Ouamri was the recipient of the Senior Award (Spectral Estimation Technical Area) from the IEEE Signal Processing Society in 1990. 